\title{
Greater Energy Cost During Standardized Walking is Associated With Diminished Fitness in Older Adults
}

Ross, AF, Schrack, JA, Dobrosielski, DA, Knuth, ND. Towson University, Towson, MD, Johns Hopkins University, Baltimore, MD

Persistently elevated walking economy, or energetic inefficiency, may lead to diminished energy reserve for physical activity. Over time this may lead to accelerated loss of function with age and development of fatigue; however, the relationship between walking economy and functional capacity in older adults is not well known. PURPOSE: To examine the relationship between walking economy and fitness in older adults.

METHODS: Walking economy was measured as the average rate of oxygen consumption over the last 2 minutes of 5 minutes of treadmill walking at 1.5 mile per hour in 50 older participants ( 23 women, aged $69 \pm 9 \mathrm{yrs}$ ) of the Towson University Longitudinal Aging Study at Towson (LAST). Aerobic fitness was assessed as time to complete 400 meters of fast-paced walking over a 20-meter course. Fatigability was defined as performance deterioration between laps and was calculated as the greatest percent increase in lap time between the second and subsequent laps. The association between the rate of oxygen composition and aerobic fitness and fatigability was modeled using linear regression, with $400 \mathrm{~m}$ walk time or fatigability as a predictor adjusting for age, fat mass, and fat-free mass. RESULTS: In the adjusted model, time to complete the $400 \mathrm{~m}$ walk (mean $273 \pm 50$ seconds) showed a significant relationship with rate of oxygen consumption $(\beta=0.6, \mathrm{p}=0.05)$, indicating that the cost of walking was $0.6 \mathrm{~mL} / \mathrm{min}$ higher for each one second increase in $400 \mathrm{~m}$ walk time. Fatigability (mean $5.8 \pm 4.1 \%$ ) also showed a significant relationship with the rate of oxygen consumption $(\beta=661, p<$ 0.05), indicating that the cost of walking was $661 \mathrm{~mL} / \mathrm{min}$ higher for each 1 percent increase in fatigability. CONCLUSION: A significant relationship occurs between poor walking economy and reduced aerobic fitness in older adults. The temporal association between reduced aerobic fitness and poor efficiency is unknown, however the combination of these two factor made lead to greater fatigability hastening the age related decline in physical activity. 